\section{Towards Clinical Bioinformatics: Redux 2015}

\author{
R. B. Altman \\ Stanford University, Stanford, CA, USA
}

In 2004, I was invited to write a preface for the IMIA Yearbook of Medical Informatics, and I was pleased to submit a paper entitled "Towards Clinical Bioinformatics." In that preface, I recognized the great (and continuing!) value of exercises that allow informatics generalists to keep track of progress in informatics broadly, and not just in their focused area of expertise. The IMIA Yearbook is one of the first resources in medical informatics to provide valuable curation of the literature, and to highlight key developments for a generalist audience. Other notable activities along these lines include annual research progress updates at large conferences, such as those that routinely occur at the fall meeting of the American Medical Informatics Association (AMIA), and the spring summit on translational bioinformatics and clinical research informatics also sponsored by AMIA. In addition, of course, the IMIA Yearbook produces reviews of published articles that appear in the archived literature.

In 2004, my preface focused on two emerging areas that were relatively new for the yearbook: bioinformatics and consumer informatics. I made three observations: (1) the mounting challenge to "translate the basic science of genomics into clinically useful tools for diagnosis and treatment," (2) the increasing use by consumers of the world wide web to manage complex information, and (3) the idea that the combination of these two would present potent challenges. Consumers might purchase medical services online and present the results to their physicians, with a request for integration of the information into their care. Imagine if patients had independent access to their own genetic information!

Since 2004, the term translational biomedical informatics (TBI) has replaced the

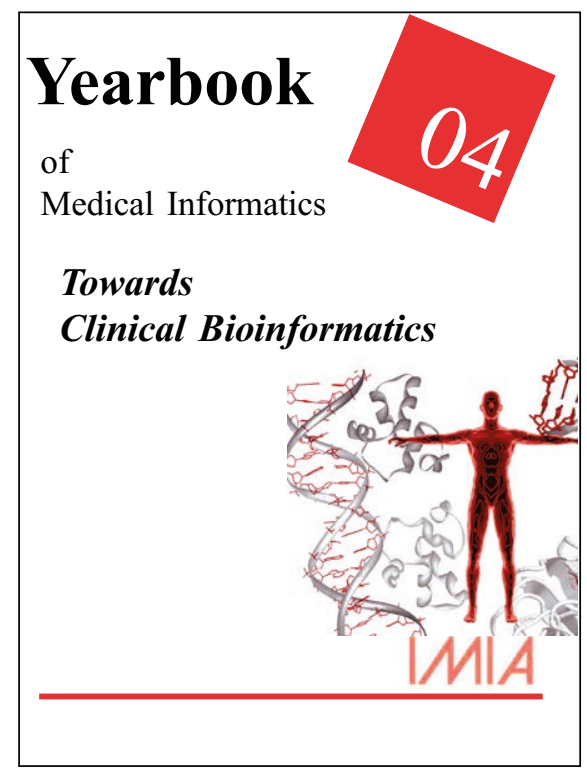

term clinical bioinformatics. TBI captures many of the key ideas and opportunities that were emerging ten years ago. Translational science has taken on a life of its own as part of many efforts in translating basic biomedical discoveries into clinically-relevant capabilities (therapeutics, diagnostics, and devices). Biomedical informatics has come to describe the creation of informatics methods that may include the biological world (including DNA, RNA, protein, small molecules, cells and how they interact over time), and the clinical world (including patients, diagnoses, signs, symptoms and how they interact over time). TBI taken together, then, is focused on the challenge of translating basic capabilities and discoveries provided by informatics methods into clinically useful tools. My characterization was overly specific; it is clear that TBI is about bringing many informatics-driven capabilities and discoveries to the point of care, and not just those based on genomics. There are great opportunities to translate basic informatics capabilities in mobile health, electronic medical record secondary use, and population health data analytics into clinical care. The intersecting worlds of translation and informatics have created a rich fabric of challenges and opportunities for the field.

The amazing growth in amount and diversity of health-related data, fueled by the internet, was difficult to anticipate. As expected, our ability to measure the genome, 
transcriptome, metabolome, and proteome has advanced and has become more comprehensive. At the same time, political, economic, and social forces conspired to accelerate the uptake of electronic medical records in the United States and worldwide. Cell phones have penetrated the global population so that they can be used to pull data for disease-surveillance and to push data for therapy and intervention. Startup companies with an interest in health and disease have created compelling disease-specific fora for patients and their caregivers to share their experiences and exchange information. Patient advocacy groups have created mechanisms to amplify the voices of their members to highlight problems that may not have been fully perceived previously. Social media such as Facebook and Twitter have created mechanisms for broadcast communication that (not surprisingly) is often relevant to health. The search logs of internet search companies have been used to infer epidemic outbreaks and drug interactions. Large cohorts have been created and the internet is a primary means of maintaining the connection with the cohort members. We, in translational biomedical informatics, have the opportunity to work in partnership with these groups to create novel methods to analyze these data, integrate information about the individual's biology with their environment and lifestyle, and help figure out how to improve the health of the public, and the health of the individuals.

The synthesis of bioinformatics and consumer challenges has arrived: It is now possible for consumers to order (via the internet) either one million genotype measurements or even their entire genome (three billion DNA base measurements). Today, they can bring this to their provider and ask for help in interpretation: They will very likely be disap- pointed. The key things we needed in 2004 and continue to need: a deep understanding of the clinical significance of our basic measurements, and information systems to present the information to practitioners in a form they can use. The "understanding challenge" begins with genomics - genomic measurements are breathtaking but are only beginning to provide actionable cost-effective information - the early victories may be in rare disease diagnosis, cancer, and optimizing drug therapy. But our need for evaluating clinical utility extends to the other modalities. How do we use mobile health data about heart rate, daily movement, and response time to improve the management and health of patients? How do we leverage huge databases of medical records to recognize the best and worst practices in current clinical care? How do we take advantage of sensing technology for early identification of disease in an individual and in a population?

The "dissemination challenge" similarly remains. Once we have reduced these powerful data sources to actionable knowledge, we must deliver decision support to health-care providers and to the patients themselves. The patients and providers need the best knowledge boiled down to actionable advice that they can understand and consider. Alarms and alerts are very unlikely to be the answer: They are interruptions (by definition exceptions) that should not be a routine part of the designed workflow.

We therefore have a more challenging task of redesigning the interaction between care providers and their decision support systems - in many ways to catch up with the immensely successful interfaces now delivered on mobile devices, smart phones, and tablets. Imagine how different from current systems an app-based environment for providers could be each clinical decision could have its own visual environment and metaphors, designed optimally to reflect the key features of that decision. Pharmacy modules could highlight drug interactions (and their level of danger) with creative manipulation of font sizes and colors. Cancer and cardiovascular risk modules could combine summaries of lifestyle risks and intrinsic genetic risks to create summaries of most likely valuable interventions, and their optimal timing. Radiological consultations could include images annotated with expert interpretation and recommended follow-up. Clinical labs could show trends over time, integrated with key clinical events, and annotated for recommended follow-up and pointers to workups that might be indicated by the trends. Each app would focus the attention of the provider on a particular realm of healthcare, and be optimized to help organize actions in that realm. Meta-apps could watch the activity over the individual activities and make sure that the key long term interventions and indicators were measured and used for appropriate action. They would ensure that the provider and the patient integrate the information from the individual apps to create overall plans and priorities to optimize patient health.

So how are we doing in all these efforts? The 2016 issue of the IMIA Annual Yearbook gives an exciting glimpse of current progress, and provides great optimism that our field will continue to step up to serve society by using informatics to improve healthcare.

\section{Correspondence to:}

Russ B. Altman, MD, PhD

Stanford University

443 Via Ortega Drive

Shriram 209, MC: 4245

Stanford, CA 94305-4145, USA

E-mail: russ.altman@stanford.edu 